\title{
ON THE LITTLEWOOD-PALEY $g$-FUNCTION AND THE LUSIN $s$-FUNCTION
}

\author{
BY \\ GEORGE GASPER, JR.(')
}

1. Introduction. Let $F(z)$ be a function regular in the unit disc $|z|<1$. In their work on Fourier series and power series, Littlewood and Paley [6] introduced the function

$$
g(F)(\theta)=\left(\int_{0}^{1}(1-r)\left|F^{\prime}\left(r e^{i \theta}\right)\right|^{2} d r\right)^{1 / 2}
$$

and proved (although they stated it in a form valid for $p>1$ only) that if $F \in H^{p}$, $p>0$, then

$$
\|g(F)\|_{p} \leqq A_{p}\|F\|_{p}
$$

The letter $A$ denotes a positive constant which is not necessarily the same at each occurrence and which, except when otherwise stated, depends only on the parameters indicated by subscripts.

In his work on boundary values of regular iunctions, Lusin [7] introduced the function

$$
s(F)(\theta)=\left(\iint_{\Omega(\theta)}\left|F^{\prime}(x+i y)\right|^{2} d x d y\right)^{1 / 2},
$$

where $\Omega(0)=\Omega$ is a standard "kite-shaped" region inside the unit disc with vertex at $z=1$ and $\Omega(\theta)$ is the region $\Omega$ rotated through an angle $\theta$ around $z=0$.

Marcinkiewicz and Zygmund [8] proved that if $F \in H^{p}, p>0$, then

$$
\|s(F)\|_{p} \leqq A_{p, \Omega}\|F\|_{p}
$$

They also demonstrated that $s$ is essentially a majorant of $g$, i.e., $g(F)(\theta) \leqq A_{\Omega} s(F)(\theta)$.

These results were extended to the class $H^{p}$ in the half-plane by Waterman [15]. The proofs given in the above-mentioned papers for (1.1) and (1.2) depend on the Blaschke product decomposition of regular functions and on the regularity of a branch of $F^{\lambda}, \lambda>0$, where $F$ is a regular function which never assumes the value

Presented to the Society, in part, January 24, 1967 under the title On the functions of Littlewood-Paley and Lusin in the unit n-sphere; received by the editors July 20, 1967.

(1) Supported in part by the National Aeronautics and Space Administration. The author wishes to thank Professor D. Waterman for suggesting the topic and for his encouragement. The author also wishes to thank Professor $\mathbf{R}$. Askey for observing a simplification at the end of \$7. Some of the results obtained in this paper were announced in [4]. 
zero. This complex variable method does not extend to higher dimensions. However, E. M. Stein [12] has extended these results to functions harmonic in the half-space with boundary values in a class $L^{p}, p>1$, by utilizing an interpolation theorem of Marcinkiewicz. In 1965, Calderón [3] gave a new proof of (1.2) for the class $H^{p}$ in the half-plane by applying Green's formula. Also in 1965, Muckenhoupt and Stein [9] extended (1.1), for $p>1$, to ultraspherical expansions by using both (1.1) and estimates for the differentiated ultraspherical Poisson kernel.

An $H^{p}$-theory for the unit sphere in $E_{n}$ can be constructed analogous to that given by E. M. Stein and G. Weiss [13] for the half-space. In this paper we shall present a method which enables us to extend (1.1) and (1.2) to both the class $H^{p}$ in the unit sphere and the class $H^{p}$ in the half-space for values of $p$ in a range reaching below 1 ; namely, $p>(n-2) /(n-1)$, where $n$ is the dimension of the space. In addition, we shall show how our method can be modified in order to extend (1.1) and (1.2) to functions which are harmonic inside the unit sphere (or in the half-space) with boundary values in a class $L^{p}, p>1$, without using the interpolation theorem of Marcinkiewicz. For the two-dimensional case, our method uses an easily proved inequality instead of the previously mentioned tools. In contrast to Calderón's technique, throughout our calculations no singularities are created by the zeros of $F$ (see [4], where we illustrate the method by presenting a proof of $(1.1)$ for $0<p \leqq 2$ ). We shall present our results for the unit sphere in Part I (\$\$2-5) and for the halfspace in Part II (\$\$ and 7).

\section{PART I. RESULTS FOR THE UNIT SPHERE}

2. Background material and main results. We shall employ the following notation. The vector $x=\left(x_{1}, x_{2}, \ldots, x_{n}\right)$ will denote a point in Euclidean $n$-space, $E_{n} ;|x|$ denotes the length of the vector $x$, i.e., $|x|=\left(x_{1}^{2}+x_{2}^{2}+\cdots+x_{n}^{2}\right)^{1 / 2} ; d x$ denotes the element of Euclidean $n$-dimensional volume; $\nabla$ and $\Delta$ denote the gradient and Laplace operators in $E_{n} ; \Sigma$ is the open unit sphere, $|x|<1$, with boundary $\partial \Sigma,|x|=1 ; \theta$ denotes a point on $\partial \Sigma$; and $d \theta$ denotes the $(n-1)$-dimensional Euclidean element of volume on $\partial \Sigma$. Thus $x=r \theta$, where $r=|x|$ and $\theta=x / r$. We shall also let $y=\left(y_{1}, y_{2}, \ldots, y_{n}\right)$ denote a point in $E_{n}$ and $\sigma$ denote a point on $\partial \Sigma$. In order to simplify notation, the dependence of general constants on the dimension will not be displayed.

We recall that in cartesian and spherical coordinates:

$$
x=r \theta=\left(x_{1}, x_{2}, \ldots, x_{n}\right)
$$

where

$$
\begin{aligned}
x_{1} & =r \sin t_{1} \cdots \sin t_{n-2} \sin t_{n-1}, \\
x_{2} & =r \sin t_{1} \cdots \sin t_{n-2} \cos t_{n-1}, \\
& \vdots \\
x_{n-1} & =r \sin t_{1} \cos t_{2}, \\
x_{n} & =r \cos t_{1},
\end{aligned}
$$


and $\left(t_{1}, t_{2}, \ldots, t_{n-1}\right)$ belongs to the set

$$
\begin{aligned}
Q=\left\{\left(t_{1}, t_{2}, \ldots, t_{n-1}\right): 0 \leqq t_{j} \leqq \pi, j=1,2, \ldots, n-2 ; 0 \leqq t_{n-1}<2 \pi\right\}, \\
d x=r^{n-1} d r d \theta,
\end{aligned}
$$

where

$$
\begin{gathered}
d \theta=\sin ^{n-2} t_{1} \sin ^{n-3} t_{2} \cdots \sin t_{n-2} d t_{1} d t_{2} \cdots d t_{n-1}, \\
|\nabla w|^{2}=\sum_{k=1}^{n}\left(\frac{\partial w}{\partial x_{k}}\right)^{2}=\left(\frac{\partial w}{\partial r}\right)^{2}+\sum_{j=1}^{n-1}\left(\frac{1}{v_{j}} \frac{\partial w}{\partial t_{j}}\right)^{2}
\end{gathered}
$$

and

$$
\Delta w=\sum_{k=1}^{n} \frac{\partial^{2} w}{\partial x_{k}^{2}}=\frac{1}{v} \frac{\partial}{\partial r}\left(v \frac{\partial w}{\partial r}\right)+\sum_{j=1}^{n-1} \frac{1}{v} \frac{\partial}{\partial t_{j}}\left(\frac{v}{v_{j}^{2}} \frac{\partial w}{\partial t_{j}}\right)
$$

where

$$
v=r^{n-1} \sin ^{n-2} t_{1} \sin ^{n-3} t_{2} \cdots \sin t_{n-2}
$$

$v_{1}=r, v_{2}=r \sin t_{1}, \ldots, v_{n-1}=r \sin t_{1} \sin t_{2} \cdots \sin t_{n-2}$.

By $L^{p}(\partial \Sigma), p>0$, we mean the class of functions $f(\theta)$ whose $p$ th power is integrable over $\partial \Sigma$. The norm in $L^{p}(\partial \Sigma)$ is defined by

$$
\|f\|_{p}=\|f(\theta)\|_{p}=\left(\int_{\partial \Sigma}|f(\theta)|^{p} d \theta\right)^{1 / p} .
$$

If $f \in L^{p}(\partial \Sigma), p \geqq 1$, then its Poisson integral $u(x), x \in \Sigma$, is given by

$$
u(x)=u(f)(x)=\frac{1}{|\partial \Sigma|} \int_{\partial \Sigma} f(\theta) \frac{1-|x|^{2}}{|\theta-x|^{n}} d \theta
$$

where $|\partial \Sigma|$ denotes the $(n-1)$-dimensional Euclidean volume of $\partial \Sigma$.

Then $u(x)$ is harmonic in $\Sigma, u(x)$ converges to $f(\theta)$ for almost every $\theta \in \partial \Sigma$ as $x$ tends nontangentially to $\theta, u(r \theta)$ converges to $f(\theta)$ in the $L^{p}(\partial \Sigma)$ norm as $r \rightarrow 1$, and $\|f\|_{p}=\lim _{r \rightarrow 1}\|u(r \theta)\|_{p}=\sup _{0 \leqq r<1}\|u(r \theta)\|_{p}$.

As an extension of the notion of a function of one complex variable regular in a region, we use a system of conjugate harmonic functions, i.e., an $n$-tuple $F(x)$ $=\left(u_{1}(x), u_{2}(x), \ldots, u_{n}(x)\right)$ of real-valued harmonic functions which, in a region, satisfy the generalized Cauchy-Riemann equations

$$
\sum_{j=1}^{n} \frac{\partial u_{j}}{\partial x_{j}}=0, \quad \frac{\partial u_{j}}{\partial x_{k}}=\frac{\partial u_{k}}{\partial x_{j}}
$$

In the two-dimensional case, it is very well known that $|F|^{p}$ is subharmonic whenever $p>0$ and, more generally, $\log |F|$ is subharmonic. For $n \geqq 2,|F(x)|^{p}$ is subharmonic whenever $p \geqq(n-2) /(n-1)$, as was shown by Stein and Weiss [13]. By employing harmonic majorants of subharmonic functions, they developed the $H^{p}$-theory for the half-space. We shall need analogous results concerning the class $H^{p}$ in $\Sigma$. 
If $F(x)$ is a system of conjugate harmonic functions in $\Sigma$, then $F(x)$ is said to belong to the class $H^{p}(\Sigma), p>0$, whenever its norm defined by

$$
\|F\|_{p}=\sup _{0 \leqq r<1}\left(\int_{\partial \Sigma}|F(r \theta)|^{p} d \theta\right)^{1 / p}
$$

is finite.

By proceeding as in [13], it can be shown that if $F \in H^{p}(\Sigma), p>(n-2) /(n-1)$, then the nontangential limit $F(\theta)=\left(u_{1}(\theta), u_{2}(\theta), \ldots, u_{n}(\theta)\right)$ exists for almost every $\theta \in \partial \Sigma, F(r \theta)$ converges to $F(\theta)$ in the $L^{p}(\partial \Sigma)$ norm as $r \rightarrow 1$, and

$$
\|F\|_{p}=\lim _{r \rightarrow 1}\|F(r \theta)\|_{p}=\|F(\theta)\|_{p}
$$

It can be shown that the nontangential limit also exists when $p=(n-2) /(n-1)$. All of the lemmas needed in the proofs are either already known for the sphere or they can be obtained by modifying known results slightly (see Aronszajn and Smith [1], Calderón [2], Privaloff [10], K. T. Smith [11], and de la Vallée Poussin [14]). We shall omit the proofs of these results (the details are contained in the authors dissertation, Wayne State University).

We now define the Littlewood-Paley $g$-function for the unit sphere by

$$
\begin{aligned}
& g(f)(\theta)=g(u)(\theta)=\left(\int_{0}^{1}(1-r)|\nabla u(r \theta)|^{2} d r\right)^{1 / 2}, \\
& g(F)(\theta)=\left(\frac{1}{n} \sum_{j=1}^{n} g^{2}\left(u_{j}\right)(\theta)\right)^{1 / 2} .
\end{aligned}
$$

For the Lusin $s$-function, the kite-shaped two-dimensional region is replaced by an open cone $\Omega_{\delta}(\theta), 0<\delta<1$, consisting of all points in $\Sigma$ which are on line segments joining $\theta$ to $|x|<\delta$.

The Lusin $s$-function for the unit sphere is then defined by

$$
\begin{aligned}
& s(f)(\theta)=s(u)(\theta)\left(\int_{\Omega_{\delta}(\theta)} \frac{|\nabla u(x)|^{2}}{(1-|x|)^{n-2}} d x\right)^{1 / 2}, \\
& s(F)(\theta)=\left(\frac{1}{n} \sum_{j=1}^{n} s^{2}\left(u_{j}\right)(\theta)\right)^{1 / 2} \cdot
\end{aligned}
$$

When $n=2$, the above definitions are the classical ones. As in the unit disc, $s$ is essentially a majorant of $g$, i.e.,

$$
g(f)(\theta) \leqq A_{\delta} s(f)(\theta), \quad g(F)(\theta) \leqq A_{\delta} s(F)(\theta) .
$$

These inequalities can easily be obtained by using the fact that $|\nabla u|^{2}$ is subharmonic and following the argument given by Marcinkiewicz and Zygmund [8] for the case $n=2$. See also Stein $[12$, p. 447].

Our extensions of (1.1) and (1.2) to the classes $H^{p}(\Sigma)$ and $L^{p}(\partial \Sigma)$ are contained in the following two theorems. 
THEOREM 1. If $F \in H^{p}(\Sigma),(n-2) /(n-1)<p<\infty$, then $\|g(F)\|_{p} \leqq A_{p}\|F\|_{p}$ and $\|s(F)\|_{p} \leqq A_{p, \delta}\|F\|_{p}$.

THEOREM 2. If $f \in L^{p}(\partial \Sigma), 1<p<\infty$, then $\|g(f)\|_{p} \leqq A_{p}\|f\|_{p}$ and $\|s(f)\|_{p} \leqq$ $A_{p, \delta}\|f\|_{p}$.

The proofs of these theorems will be split into two parts; in $\$ 4$ we consider the case when $p$ assumes values $\leqq 2$ and in $\S 5$ we consider the case when $p>2$. In $\S 3$ we present our main tools and some basic lemmas.

3. Preliminary lemmas. The first two lemmas are the main tools of our method.

LEMMA 1. Let $c>0$ and $(n-2) /(n-1)<p \leqq 2$. Then, for any system of conjugate harmonic functions $F(x)$,

$$
2 \sum_{k=1}^{n}\left|\nabla u_{k}\right|^{2}=\Delta\left(|F|^{2}\right) \leqq \frac{2 n}{p(p n-p-n+2)}\left(|F|^{2}+c\right)^{(2-p) / 2} \Delta\left(\left(|F|^{2}+c\right)^{p / 2}\right) .
$$

LEMMA 2. Let $c>0$ and $1<p \leqq 2$. Then, for any real-valued harmonic function $u(x)$,

$$
2|\nabla u|^{2}=\Delta\left(u^{2}\right) \leqq \frac{2}{p(p-1)}\left(u^{2}+c\right)^{(2-p) / 2} \Delta\left(\left(u^{2}+c\right)^{p / 2}\right) .
$$

The Laplacians are nonnegative and, since $c>0$, they exist at each point in the domain of definition of the function ( $F$ or $u$ ), even those where the function is zero. Hence, there are no singularities to be concerned with at the zeros of the function.

Proof of Lemma 1. In the following calculations, if $G=\left(h_{1}, \ldots, h_{n}\right)$ is another vector function, we let

$$
F \cdot G=u_{1} h_{1}+\cdots+u_{n} h_{n} \text { and } G_{x_{k}}=\left(\partial h_{1} / \partial x_{k}, \ldots, \partial h_{n} / \partial x_{k}\right) .
$$

We shall also let $w(x)=|F(x)|^{2}+c$. It is easy to verify that

$$
\begin{gathered}
\frac{\partial}{\partial x_{k}}|F|^{2}=2\left(F \cdot F_{x_{k}}\right), \quad \frac{\partial^{2}}{\partial x_{k}^{2}}|F|^{2}=2\left|F_{x_{k}}\right|^{2}+2\left(F \cdot F_{x_{k} x_{k}}\right), \\
\frac{\partial^{2}}{\partial x_{k}^{2}}\left(w^{p / 2}\right)=\frac{p(p-2)}{4} w^{(p-4) / 2}\left(\frac{\partial}{\partial x_{k}}|F|^{2}\right)^{2}+\frac{p}{2} w^{(p-2) / 2} \frac{\partial^{2}}{\partial x_{k}^{2}}|F|^{2} .
\end{gathered}
$$

Since the components of $F$ are harmonic, a summation over $k$ yields

$$
\Delta\left(w^{p / 2}\right)=p(p-2) w^{(p-4) / 2} \sum_{k=1}^{n}\left(F \cdot F_{x_{k}}\right)^{2}+p w^{(p-2) / 2} \sum_{k=1}^{n}\left|F_{x_{k}}\right|^{2} .
$$

Stein and Weiss $[13$, p. 34$]$ have shown that

$$
\sum_{k=1}^{n}\left(F \cdot F_{x_{k}}\right)^{2} \leqq \frac{n-1}{n}|F|^{2} \sum_{k=1}^{n}\left|F_{x_{k}}\right|^{2} .
$$


From (3.1) and (3.2), we have $(p(2-p) \geqq 0)$

$$
p w^{(p-2) / 2} \sum_{k=1}^{n}\left|F_{x_{k}}\right|^{2} \leqq \Delta\left(w^{p / 2}\right)+\frac{p(2-p)(n-1)}{n} w^{(p-2) / 2} \sum_{k=1}^{n}\left|F_{n_{k}}\right|^{2}
$$

which reduces to

$$
p(p n-p-n+2) w^{(p-2) / 2} \sum_{k=1}^{n}\left|F_{x_{k}}\right|^{2} \leqq n \Delta\left(w^{p / 2}\right) .
$$

Lemma 1 now follows from the above inequality and the observation that

$$
\Delta\left(|F|^{2}\right)=2 \sum_{k=1}^{n}\left|F_{x_{k}}\right|^{2}=2 \sum_{k=1}^{n}\left|\nabla u_{k}\right|^{2}
$$

Proof of Lemma 2. In the proof of Lemma 1 , replace $F(x)$ by $(u(x), 0, \ldots, 0)$ and use the identity

in place of (3.2).

$$
\sum_{k=1}^{n}\left(u \frac{\partial u}{\partial x_{k}}\right)^{2}=u^{2} \sum_{k=1}^{n}\left(\frac{\partial u}{\partial x_{k}}\right)^{2}
$$

The next three lemmas are generalizations of some very well-known results of Hardy and Littlewood [5].

LEMMA 3. For $f \in L^{p}(\partial \Sigma), p \geqq 1$, define

$$
M(f)(\theta)=\sup \frac{1}{|C(\theta, r)|} \int_{C(\theta, r)}|f(\sigma)| d \sigma,
$$

where the supremum is taken over all spherical caps $C(\theta, r)=\{\sigma:|\sigma-\theta|<r\}$ and $|C(\theta, r)|$ denotes the $(n-1)$-dimensional volume of $C(\theta, r)$. If $p>1$, then

$$
\|M(f)\|_{p} \leqq A_{p}\|f\|_{p} .
$$

LEMMA 4. Let $w(x), x \in \Sigma$, be a nonnegative subharmonic function, and let

If $p>1$, then

$$
N(w)(\theta)=N_{\delta}(w)(\theta)=\sup \left\{w(x): x \in \Omega_{\delta}(\theta)\right\} .
$$

$$
\|N(w)\|_{p} \leqq A_{p, \delta} \sup _{0 \leqq r<1}\|w(r \theta)\|_{p} .
$$

Lemma 5. Suppose that $F \in H^{p}(\Sigma), p>(n-2) /(n-1)$, and let

Then

$$
N(F)(\theta)=N_{\delta}(F)(\theta)=\sup \left\{|F(x)|: x \in \Omega_{\delta}(\theta)\right\} .
$$

$$
\|N(F)\|_{p} \leqq A_{p, \delta}\|F\|_{p}
$$

Lemma 3 is a special case of a theorem proved for more general domains by K. T. Smith [11, Theorem 1]. Lemma 4 can be obtained by a simple modification of the proof which K. T. Smith [11] gave for his Theorem 6. Since $|F(x)|^{p}$ is subharmonic whenever $p \geqq(n-2) /(n-1)$, Lemma 5 is an immediate consequence of Lemma 4. 
The proofs of Theorems 1 and 2 also require

LEMMA 6. Suppose that $F(x)=\left(u_{1}(x), \ldots, u_{n}(x)\right), x \in \Sigma$, is an $n$-tuple of realvalued harmonic functions (not necessarily satisfying (2.4)). Let

$$
c=c(\delta)=\sup \left\{|F(x)|^{2}:|x| \leqq(\delta+1) / 2\right\},
$$

where $\delta$ is fixed so that $0<\delta<1$. If $p>0$ and $|x| \leqq \delta$, then

$$
\begin{aligned}
\left|(\partial / \partial r) u_{j}(x)\right| \leqq\left|\nabla u_{j}(x)\right| & \leqq A_{\delta} c^{1 / 2}, \quad j=1,2, \ldots, n \\
\left|(\partial / \partial r)\left(|F(x)|^{2}+c\right)^{p / 2}\right| & \leqq A_{p, \delta} c^{p / 2}, \\
\left|\Delta\left(\left(|F(x)|^{2}+c\right)^{p / 2}\right)\right| & \leqq A_{p, \delta} c^{p / 2} .
\end{aligned}
$$

Proof. Integrating $\partial u_{j} / \partial x_{k}$ over the interior of the sphere with center $x$ and radius $R=(1-\delta) / 2$, applying Green's theorem, and using the mean value property of harmonic functions, we get (3.3). Then (3.4) follows directly from (3.3), and (3.5) follows directly from (3.1) and (3.3).

4. The cases $H^{p}(\Sigma),(n-2) /(n-1)<p \leqq 2$, and $L^{p}(\partial \Sigma), 1<p \leqq 2$. We first present the following:

Proof of Theorem 1; case when $(n-2) /(n-1)<p \leqq 2$. Due to (2.5), it suffices to show that $\|s(F)\|_{p} \leqq A_{p, \delta}\|F\|_{p}$.

Let $F_{R}(x)=F(R x), 0<R<1$. If we had the inequality $\left\|S\left(F_{R}\right)\right\|_{p} \leqq A_{p, \delta}\left\|F_{R}\right\|_{p}$, then, by Fatou's lemma, since $s(F)(\theta) \leqq \lim \inf _{R \rightarrow 1} s\left(F_{R}\right)(\theta)$, we would have

$$
\|s(F)\|_{p} \leqq \liminf _{R \rightarrow 1}\left\|s\left(F_{R}\right)\right\|_{p} \leqq \lim _{R \rightarrow 1} A_{p, \delta}\left\|F_{R}\right\|_{p}=A_{p, \delta}\|F\|_{p}
$$

the general result. Hence we may assume that $F(x)$ is a system of conjugate harmonic functions for $|x| \leqq 1$.

We write

$$
\begin{gathered}
\Omega(\theta)=\Omega_{\delta}(\theta), \quad s=s(F), \quad d=(\delta+1) / 2, \quad r=|x|, \\
N(\theta)=\sup _{x \in \Omega_{d}(\theta)}|F(x)|, \quad c=\sup _{|x| \leqq d}|F(x)|^{2}, \quad w(x)=\left(|F(x)|^{2}+c\right)^{p / 2} .
\end{gathered}
$$

Application of Lemma 1 gives

$$
\begin{aligned}
s^{2}(\theta) & \leqq A_{\mathcal{p}} \int_{\Omega(\theta)} \frac{\left(|F|^{2}+c\right)^{(2-p) / 2} \Delta w}{(1-r)^{n-2}} d x \\
& \leqq A_{p} N^{2-p}(\theta) \int_{\Omega(\theta)} \frac{\Delta w}{(1-r)^{n-2}} d \dot{x} .
\end{aligned}
$$

Hence, by Hölder's inequality,

$$
\|s\|_{p} \leqq A_{p}\|N\|_{p}^{(2-p) / 2}\left(\int_{\partial \Sigma} \int_{\Omega(\theta)} \frac{\Delta w}{(1-r)^{n-2}} d x d \theta\right)^{1 / 2} .
$$


An elementary argument shows that if $\chi_{\theta}(x)$ is the characteristic function of $\Omega(\theta)$ and

then

$$
J_{r}=\int_{\partial \Sigma} \chi_{\theta}(r \sigma) d \theta
$$

$$
J_{r} \leqq A_{\delta}(1-r)^{n-1}
$$

Applying (4.2), we have

$$
\begin{aligned}
\int_{\partial \Sigma} \int_{\Omega(\theta)} \frac{\Delta w}{(1-r)^{n-2}} d x d \theta & =\int_{\partial \Sigma} \int_{\Sigma} \frac{\chi_{\theta}(x) \Delta w}{(1-r)^{n-2}} d x d \theta=\int_{\Sigma} \frac{J_{r} \Delta w}{(1-r)^{n-2}} d x \\
& \leqq A_{\delta} \int_{\partial \Sigma} \int_{0}^{1}(1-r) \Delta w(r \theta) d r d \theta=A_{\delta}\left\|G_{p}^{2}\right\|_{1},
\end{aligned}
$$

where

We prove now that

$$
G_{p}^{2}(\theta)=\int_{0}^{1}(1-r) \Delta w(r \theta) d r
$$

$$
\left\|G_{p}^{2}\right\|_{1} \leqq A_{p, \delta}\|N\|_{p}^{p}
$$

Letting

$$
G_{p}^{2}(\theta)=\int_{0}^{\delta}+\int_{\delta}^{1}=I_{1}^{2}+I_{2}^{2}
$$

reduces the proof of (4.4) to showing that

$$
\left\|I_{k}^{2}\right\|_{1} \leqq A_{p, \delta}\|N\|_{p}^{p}, \quad k=1,2 .
$$

By Lemma 6 and the observation that $c \leqq N^{2}(\theta)$ for all $\theta$, we get

$$
\left\|I_{1}^{2}\right\|_{1} \leqq A_{p, \delta}\left\|\int_{0}^{\delta}(1-r) c^{p / 2} d r\right\|_{1} \leqq A_{p, \delta}\|N\|_{p}^{p}
$$

which gives (4.5) for $I_{1}$.

For $I_{2}$, we use (2.3) to write

$$
\begin{aligned}
I_{2}^{2} & =\int_{0}^{1} \frac{1-r}{v} \frac{\partial}{\partial r}\left(v \frac{\partial w}{\partial r}\right) d r+\sum_{j=1}^{n-1} \int_{\delta}^{1} \frac{1-r}{v} \frac{\partial}{\partial t_{j}}\left(\frac{v}{v_{j}^{2}} \frac{\partial w}{\partial t_{j}}\right) d r \\
& =P+\sum_{j=1}^{n-1} P_{j} .
\end{aligned}
$$

Let us first consider $P$. Integrating by parts ( $F$ being a system of conjugate harmonic functions for $|x| \leqq 1$ ) and applying Lemma 6 , we obtain

$$
\begin{aligned}
P & =\int_{\delta}^{1} \frac{1-r}{r^{n-1}} \frac{\partial}{\partial r}\left(r^{n-1} \frac{\partial w}{\partial r}\right) d r \\
& =\left[(1-r) \frac{\partial w}{\partial r}\right]_{\delta}^{1}+\left[\left(2-n+\frac{n-1}{r}\right) w\right]_{\delta}^{1}+(n-1) \int_{\delta}^{1} \frac{w}{r^{2}} d r \\
& \leqq A_{p, \delta} N^{p} .
\end{aligned}
$$


For $P_{j}$, an integration with respect to $t_{j}$ shows that ( $Q$ as defined in (2.1))

$$
\int_{\partial \Sigma} P_{j}(\theta) d \theta=\int_{Q} \int_{\delta}^{1} \frac{1-r}{r^{n-1}} \frac{\partial}{\partial t_{j}}\left(\frac{v}{v_{j}^{2}} \frac{\partial w}{\partial t_{j}}\right) d r d t_{1} \cdots d t_{n-1}=0
$$

because

$$
\left[\sin ^{n-j-1} t_{j} \frac{\partial w}{\partial t_{j}}\right]_{t_{j}=0}^{t_{j}=\pi}=0, \quad j<n-1
$$

and, due to periodicity,

$$
\left[\frac{\partial w}{\partial t_{n-1}}\right]_{t_{n-1}=0}^{t_{n-1}=2 \pi}=0 .
$$

Combining (4.6), (4.7), and (4.8), we get

$$
\int_{\partial \Sigma} I_{2}^{2}(\theta) d \theta \leqq A_{p, \delta}\|N\|_{p}^{p}
$$

which gives (4.5) for $I_{2}$. This concludes the proof of (4.4).

From (4.1), (4.3), (4.4), and Lemma 5, we get

$$
\|s\|_{p} \leqq A_{p, \delta}\|N\|_{p}^{(2-p) / 2}\left\|G_{p}^{2}\right\|_{1}^{1 / 2} \leqq A_{p, \delta}\|N\|_{p} \leqq A_{p, \delta}\|F\|_{p}
$$

the desired result.

Proof of Theorem 2; case when $1<p \leqq 2$. The proof here runs along essentially the same lines as the above. We replace Lemma 1 by Lemma 2 and Lemma 5 by Lemma 4 with $w(x)=|u(f)(x)|$.

5. Theorems 1 and 2; case when $p>2$. The following lemma enables us to derive the inequalities in Theorems 1 and 2 involving the $s$-function from those for the $g$-function when $p \geqq 2$.

LEMMA 7. If $p \geqq 2$ and $u(x)$ is a function harmonic in $\Sigma$, then

$$
\|s(u)\|_{p} \leqq A_{p, \delta}\|g(u)\|_{p} .
$$

Proof. Let $s=s(u)$ and $g=g(u)$. We may write

$$
\|s\|_{p}^{2}=\left(\int_{\partial \Sigma}\left(s^{2}(\theta)\right)^{p / 2} d \theta\right)^{2 / p}=\sup _{h} \int_{\partial \Sigma} s^{2}(\theta) h(\theta) d \theta
$$

the supremum being taken over all nonnegative functions $h(\theta)$ which satisfy $\|h\|_{q} \leqq 1$, where $1 /(p / 2)+1 / q=1$.

Then, using (4.2), Lemma 3 , and the notation associated with them, we have $(x=r \sigma)$

$$
\begin{aligned}
\int_{\partial \Sigma} s^{2}(\theta) h(\theta) d \theta & =\int_{\partial \Sigma} \int_{\Sigma} \frac{h(\theta) \chi_{\theta}(x)|\nabla u(x)|^{2}}{(1-r)^{n-2}} d x d \theta \\
& =\int_{\Sigma} \frac{J_{r}|\nabla u(x)|^{2}}{(1-r)^{n-2}}\left(\frac{1}{J_{r}} \int_{\partial \Sigma} h(\theta) \chi_{\theta}(r \sigma) d \theta\right) d x \\
& \leqq \int_{\Sigma} \frac{J_{r}|\nabla u(x)|^{2} M(h)(\sigma)}{(1-r)^{n-2}} d x \\
& \leqq A_{\delta} \int_{\partial \Sigma} \int_{0}^{1}(1-r)|\nabla u(r \theta)|^{2} M(h)(\theta) d r d \theta \\
& \leqq A_{\delta}\|g\|_{p}^{2}\|M(h)\|_{q} \leqq A_{p, \delta}\|g\|_{p}^{2}\|h\|_{q} \leqq A_{p, \delta}\|g\|_{p}^{2},
\end{aligned}
$$

which, with (5.2), yields (5.1). 
We also need the next lemma, which contains generalizations of some well-known inequalities that were employed by Littlewood and Paley [6, II]. Since the proof is a relatively direct and tedious modification of that sketched for Lemma 6, it is omitted.

LEMMA 8. Suppose that $F(x)$ is a system of conjugate harmonic functions in $\Sigma$. Let

$$
c=\sup _{|x| \leqq 1 / 2}|F(x)|^{2} \quad \text { and } \quad N(\theta)=N(F)(\theta)=\sup _{x \in \Omega_{1 / 2}(\theta)}|F(x)| .
$$

If $(n-2) /(n-1)<p \leqq 2$ and $x=r \theta$, then $(j, k=1,2, \ldots, n)$

$$
\begin{gathered}
\left|\frac{\partial}{\partial r} u_{j}(x)\right| \leqq\left|\nabla u_{j}(x)\right| \leqq \frac{A N(\theta)}{1-r}, \\
\left|\nabla\left(\left(|F(x)|^{2}+c\right)^{p / 2}\right)\right| \leqq \frac{A_{p} N^{p}(\theta)}{1-r}, \\
\left|\nabla\left(\frac{\partial}{\partial x_{k}} u_{j}(x)\right)\right| \leqq \frac{A N(\theta)}{(1-r)^{2}}, \\
\left|\nabla\left(\left(|F(x)|^{2}+c\right)^{p / 2}\right)\right| \leqq A_{p} N^{p / 2}(\theta)\left[\Delta\left(\left(|F(x)|^{2}+c\right)^{p / 2}\right)\right]^{1 / 2}, \\
\left|\nabla\left[\Delta\left(\left(|F(x)|^{2}+c\right)^{p / 2}\right)\right]\right| \leqq A_{p} N^{p / 2}(\theta)\left[\Delta\left(\left(|F(x)|^{2}+c\right)^{p / 2}\right)\right]^{1 / 2} /(1-r)^{2} .
\end{gathered}
$$

We now complete the proofs of Theorems 1 and 2.

Proof of Theorem 1; case when $p>2$. In view of Lemma 7 and the inequalities

$$
s(F) \leqq \sum_{k=1}^{n} s\left(u_{k}\right), \quad g\left(u_{k}\right) \leqq n^{1 / 2} g(f),
$$

it is enough to prove that

$$
\|g(F)\|_{p} \leqq A_{p}\|F\|_{p}
$$

Moreover, due to an observation made in $\S 4$, we may assume that $F(x)$ is a system of conjugate harmonic functions for $|x| \leqq 1$.

Letting

$$
g^{2}(F)(\theta)=\int_{0}^{1 / 4}+\int_{1 / 4}^{1}=I_{1}^{2}+I_{2}^{2}
$$

reduces the problem to showing that

$$
\left\|I_{j}\right\|_{p} \leqq A_{p}\|F\|_{p}, \quad j=1,2 .
$$

Let $c$ and $N(\theta)$ be defined as in Lemma 8. Then, by (5.3) and Lemma 5,

$$
\left\|I_{1}\right\|_{p} \leqq A_{p}\|N\|_{p} \leqq A_{p}\|F\|_{p}
$$

which gives (5.9) for $I_{1}$. 
In proving (5.9) for $I_{2}$, we shall follow a procedure which is closely akin to that employed by Littlewood and Paley [6, II, pp. 60-63] for their $g$-function when $p$ is an even integer.

Let us first consider the special case $2<p \leqq 4$ (actually the following argument is valid for the wider range $(2 n-4) /(n-1)<p \leqq 4)$. Application of Lemma 1 with $p$ replaced by $p / 2$ and $w(r \theta)=\left(|F(r \theta)|^{2}+c\right)^{p / 4}$ gives

$$
\begin{aligned}
\left\|I_{2}\right\|_{p} & \leqq A_{p}\left(\int_{\partial \Sigma} N^{p(4-p) / 4}\left\{\int_{1 / 4}^{1}(1-r) \Delta w d r\right\}^{p / 2} d \theta\right)^{1 / p} \\
& \leqq A_{p}\|N\|_{p}^{(4-p) / 4}\left\|\int_{1 / 4}^{1}(1-r) \Delta w d r\right\|_{2}^{1 / 2}
\end{aligned}
$$

since $(4-p) / 4+p / 4=1$. Writing $w_{m}=w\left(r_{m}, \theta\right), m=1,2$, we see that

$$
\left\|\int_{1 / 4}^{1}(1-r) \Delta w d r\right\|_{2}^{2}=2 \int_{\partial \Sigma} \int_{1 / 4}^{1} \int_{r_{2}}^{1}\left(1-r_{1}\right)\left(1-r_{2}\right) \Delta w_{1} \Delta w_{2} d r_{1} d r_{2} d \theta
$$

Substituting for $\Delta w_{1}$ its form in spherical coordinates (using the notation in (2.3) with $r$ replaced by $r_{1}$ ) and observing that, due to Lemma 8 ,

$$
\begin{aligned}
\int_{r_{2}}^{1} \frac{1-r}{r^{n-1}} \frac{\partial}{\partial r}\left(r^{n-1} \frac{\partial w}{\partial r}\right) d r= & {\left[(1-r) \frac{\partial w}{\partial r}\right]_{r_{2}}^{1}+\left[\left(2-n+\frac{n-1}{r}\right) w\right]_{r_{2}}^{1} } \\
& +(n-1) \int_{r_{2}}^{1} \frac{w}{r_{2}} d r \leqq A_{p} N^{p / 2}
\end{aligned}
$$

whenever $1 / 4 \leqq r_{2} \leqq 1$, we obtain

$$
\begin{aligned}
\left\|\int_{1 / 4}^{1}(1-r) \Delta w d r\right\|_{2}^{2} \leqq & A_{p} \int_{\partial \Sigma} \int_{1 / 4}^{1}\left(1-r_{2}\right) N^{p / 2} \Delta w_{2} d r_{2} d \theta \\
& +2 \sum_{j=1}^{n-1} \int_{\partial \Sigma} \int_{1 / 4}^{1} \int_{r_{2}}^{1}\left(1-r_{1}\right)\left(1-r_{2}\right) \Delta w_{2} \\
& \times \frac{1}{v} \frac{\partial}{\partial t_{j}}\left(\frac{v}{v_{j}^{2}} \frac{\partial w_{1}}{\partial t_{j}}\right) d r_{1} d r_{2} d \theta \\
= & P+\sum_{j=1}^{n-1} P_{j} .
\end{aligned}
$$

Then, using Hölder's inequality,

$$
P \leqq A_{p}\|N\|_{p}^{p / 2}\left\|\int_{1 / 4}^{1}(1-r) \Delta w d r\right\|_{2}
$$

Also, an integration by parts with respect to $t_{j}$ yields

$$
P_{j}=-2 \int_{\partial \Sigma} \int_{1 / 4}^{1} \int_{r_{2}}^{1}\left(1-r_{1}\right)\left(1-r_{2}\right) \frac{1}{v_{j}^{2}}\left(\frac{\partial}{\partial t_{j}} \Delta w_{2}\right) \frac{\partial w_{1}}{\partial t_{j}} d r_{1} d r_{2} d \theta
$$


Therefore, using (5.6) and (5.7) with $p$ replaced by $p / 2$,

$$
\begin{aligned}
P_{j} & \leqq A_{p} \int_{\partial \Sigma} \int_{1 / 4}^{1} \int_{r_{2}}^{1}\left(\frac{1-r_{1}}{1-r_{2}}\right) N^{p / 2}\left(\Delta w_{1}\right)^{1 / 2}\left(\Delta w_{2}\right)^{1 / 2} d r_{1} d r_{2} d \theta \\
& \leqq A_{p} \int_{\partial \Sigma} N^{p / 2} \int_{1 / 4}^{1} \int_{r_{2}}^{1}\left[\Delta w_{2}+\left(\frac{1-r_{1}}{1-r_{2}}\right)^{2} \Delta w_{1}\right] d r_{1} d r_{2} d \theta \\
& =A_{p} \int_{\partial \Sigma} N^{p / 2}\left\{\int_{1 / 4}^{1}\left(\Delta w_{2} \int_{r_{2}}^{1} d r_{1}\right) d r_{2}\right. \\
& \left.\leqq \int_{1 / 4}^{1}\left(1-r_{1}\right)^{2} \Delta w_{1}\left(\int_{1 / 4}^{r_{1}} \frac{d r_{2}}{\left(1-r_{2}\right)^{2}}\right) d r_{1}\right\} d \theta \\
& \left.\leqq A_{p}\|N\|_{\partial \Sigma} N_{p}^{p / 2}\left\|\int_{1 / 4}^{1}(1-r) \Delta w d r\right\|_{2}^{1}(1-r) \Delta w d r\right) d \theta
\end{aligned}
$$

From (5.11), (5.12), and (5.13), we get

$$
\left\|\int_{1 / 4}^{1}(1-r) \Delta w d r\right\|_{2} \leqq A_{p}\|N\|_{p}^{p / 2}
$$

and so, due to (5.10) and Lemma 5,

$$
\left\|I_{2}\right\|_{p} \leqq A_{p}\|N\|_{p}^{(4-p) / 4}\|N\|_{p}^{p / 4} \leqq A_{p}\|F\|_{p}, \quad 2<p \leqq 4 .
$$

For the case when $2(k-1)<p \leqq 2 k, k=2,3, \ldots$, application of Lemma 1 with $p$ replaced by $p / k, c$ defined as above, and $w(r \theta)=\left(|F(r \theta)|^{2}+c\right)^{p / 2 k}$ gives

$$
\left\|I_{2}\right\|_{p} \leqq A_{p}\|N\|_{p}^{(2 k-p) / 2 k}\left\|\int_{1 / 4}^{1}(1-r) \Delta w d r\right\|_{k}^{1 / 2},
$$

since $(2 k-p) / 2 k+p / 2 k=1$.

Writing $w_{m}=w\left(r_{m} \theta\right), m=1,2, \ldots, k$, we see that

$$
\left\|\int_{1 / 4}^{1}(1-r) \Delta w d r\right\|_{k}^{k}=A_{k} \int_{\partial \Sigma} \int_{1 / 4 \leqq r_{k} \leqq \cdots \leqq r_{1} \leqq 1} \cdots \int \prod_{1}^{k}\left(1-r_{m}\right) \Delta w_{m} \prod_{1}^{k} d r_{m} d \theta \text {. }
$$

Now we substitute for $\Delta w_{1}$ its form in spherical coordinates and notice that, just as in (5.12), the integral containing the partials with respect to $r_{1}$ is not greater than

$$
A_{p}\|N\|_{p}^{p / k}\left\|\int_{1 / 4}^{1}(1-r) \Delta w d r\right\|_{k}^{k-1}
$$

Next, as in the case $k=2$, the integral ( $j$ considered fixed)

$$
\int_{\partial \Sigma} \int_{1 / 4 \leqq \ldots \leqq r_{1} \leqq 1} \cdots \int \prod_{1}^{k}\left(1-r_{m}\right) \prod_{2}^{k} \Delta w_{m} \frac{1}{v} \frac{\partial}{\partial t_{j}}\left(\frac{v}{v_{j}^{2}} \frac{\partial w_{1}}{\partial t_{j}}\right) \prod_{1}^{k} d r_{m} d \theta
$$

is integrated by parts with respect to $t_{j}$ in order to show that it equals

$$
-\int_{\partial \Sigma} \int_{1 / 4 \leqq \ldots \leqq r_{1} \leqq 1} \cdots \int v_{j}^{-2} \prod_{1}^{k}\left(1-r_{m}\right) \frac{\partial}{\partial t_{j}}\left(\prod_{2}^{k} \Delta w_{m}\right) \frac{\partial w_{1}}{\partial t_{j}} \prod_{1}^{k} d r_{m} d \theta .
$$


Then we substitute

$$
\frac{\partial}{\partial t_{j}}\left(\prod_{2}^{k} \Delta w_{m}\right)=\sum_{i=2}^{k} \frac{\partial \Delta w_{i}}{\partial t_{j}}\left(\prod_{2, m \neq i}^{k} \Delta w_{m}\right)
$$

into the above integral and consider the term ( $i$ and $j$ considered fixed)

$$
-\int_{\partial \Sigma} \int_{1 / 4 \leqq \cdots ⿱_{1} \leqq 1} \cdots \int v_{j}^{-2} \prod_{1}^{k}\left(1-r_{m}\right) \frac{\partial w_{1}}{\partial t_{j}} \frac{\partial \Delta w_{i}}{\partial t_{j}} \prod_{2, m \neq i}^{k} \Delta w_{m} \prod_{1}^{k} d r_{m} d \theta .
$$

Applying Lemma 8 with $p$ replaced by $p / k$ and proceeding as in (5.13), we find that this term is less in absolute value than

$$
\begin{aligned}
& A_{p} \int_{\partial \Sigma} N^{p / k} \int_{1 / 4 \leqq \cdots \leqq r_{1} \leqq 1} \cdots \int\left(\frac{1-r_{1}}{1-r_{i}}\right)\left(\Delta w_{1}\right)^{1 / 2}\left(\Delta w_{i}\right)^{1 / 2} \prod_{2, m \neq i}^{k}\left(1-r_{m}\right) \Delta w_{m} \prod_{1}^{k} d r_{m} d \theta \\
& \leqq A_{p} \int_{\partial \Sigma} N^{p / k}\left(\iint_{0 \leqq r_{i} \leqq r_{1} \leqq 1}\left(\frac{1-r_{1}}{1-r_{i}}\right)\left(\Delta w_{1}\right)^{1 / 2}\left(\Delta w_{i}\right)^{1 / 2} d r_{1} d r_{i}\right) \\
& \cdot\left(\int_{0 \leqq \cdots \leqq r_{i+1} \leqq r_{i-1} \leqq \cdots \leqq r_{2} \leqq 1} \cdots \int \prod_{2, m \neq i}^{k}\left(1-r_{m}\right) \Delta w_{m} \prod_{2, m \neq i}^{k} d r_{m}\right) d \theta \\
& \leqq A_{p} \int_{\partial \Sigma} N^{p / k}\left(\int_{1 / 4}^{1}(1-r) \Delta w d r\right)^{k-1} d \theta \\
& \leqq A_{p}\|N\|_{p}^{p / k}\left\|\int_{1 / 4}^{1}(1-r) \Delta w d r\right\|_{k}^{k-1}
\end{aligned}
$$

Combining these estimates, we obtain

$$
\left\|\int_{1 / 4}^{1}(1-r) \Delta w d r\right\|_{k} \leqq A_{p}\|N\|_{p}^{p / k}
$$

and so, from (5.14),

$$
\left\|I_{2}\right\|_{p} \leqq A_{p}\|N\|_{p}^{(2 k-p) / 2 k}\|N\|_{p}^{p / 2 k} \leqq A_{p}\|F\|_{p},
$$

which gives (5.9) for $I_{2}$. This completely proves Theorem 1 .

Proof of Theorem 2; case when $p>2$. In the above proof, replace the lemmas regarding systems of conjugate harmonic functions by the analogous results for a single harmonic function.

\section{PART II. RESUlTS FOR THE HALF-SPACE}

6. Background material and main results. For points in $E_{n}$ we shall continue to use the notation introduced in $\$ 2$, unless otherwise stated. A point in the halfspace, $E_{n} \times(0, \infty)$, will be denoted by $(x ; t)=\left(x_{1}, \ldots, x_{n} ; t\right)$, where $t>0 ;|(x ; t)|$ $=\left(x_{1}^{2}+\cdots+x_{n}^{2}+t^{2}\right)^{1 / 2}$; and $\nabla$ and $\Delta$ denote the gradient and Laplace operators in $E_{n+1}$. 
By $L^{p}\left(E_{n}\right), p>0$, we mean the class of functions $f(x)$ whose $p$ th power is integrable over $E_{n}$. The norm in $L^{p}\left(E_{n}\right)$ is defined by

$$
\|f\|_{p}=\|f(x)\|_{p}=\left(\int_{E_{n}}|f(x)|^{p} d x\right)^{1 / p} .
$$

If $f \in L^{p}\left(E_{n}\right), p \geqq 1$, then its Poisson integral $u(x ; t), t>0$, is given by

$$
u(x ; t)=u(f)(x ; t)=\pi^{-(n+1) / 2} \Gamma\left(\frac{n+1}{2}\right) t \int_{E_{n}} \frac{f(x-y) d y}{\left(|y|^{2}+t^{2}\right)^{(n+1) / 2}} .
$$

Then $u(x ; t)$ is harmonic in $E_{n} \times(0, \infty), f(x)=\lim _{t \rightarrow 0} u(x ; t)$ both in the $L^{p}\left(E_{n}\right)$ norm and almost everywhere, and $\|f\|_{p}=\lim _{t \rightarrow 0}\|u(x ; t)\|_{p}=\sup _{t>0}\|u(x ; t)\|_{p}$.

A system of conjugate harmonic functions in the half-space is an $(n+1)$-tuple $F(x ; t)=\left(u_{1}(x ; t), \ldots, u_{n+1}(x ; t)\right)$ of real-valued harmonic functions satisfying, in $t>0$, the generalized Cauchy-Riemann equations

$$
\frac{\partial u_{n+1}}{\partial t}+\sum_{j=1}^{n} \frac{\partial u_{j}}{\partial x_{j}}=0, \quad \frac{\partial u_{n+1}}{\partial x_{j}}=\frac{\partial u_{j}}{\partial t}, \quad \frac{\partial u_{j}}{\partial x_{k}}=\frac{\partial u_{k}}{\partial x_{j}}
$$

where $j, k=1,2, \ldots, n$. Since $F(x ; t)$ has $(n+1)$-components, $|F(x ; t)|^{p}$ is subharmonic whenever

$$
p \geqq \frac{n-1}{n}=\frac{(n+1)-2}{(n+1)-1}
$$

If $F(x ; t)$ is a system of conjugate harmonic functions in the half-space, then $F(x ; t)$ is said to belong to the class $H^{p}\left(E_{n} \times(0, \infty)\right), p>0$, whenever its norm defined by

$$
\|F\|_{p}=\sup _{0<t<\infty}\left(\int_{E_{n}}|F(x ; t)|^{p} d x\right)^{1 / p}
$$

is finite.

Stein and Weiss [13] have shown that if $F \in H^{p}\left(E_{n} \times(0, \infty)\right), p>(n-1) / n$, then the nontangential limit $F(x ; 0)=\left(u_{1}(x ; 0), \ldots, u_{n+1}(x ; 0)\right)$ exists for almost every $x \in E_{n}, F(x ; t)$ converges to $F(x ; 0)$ in the $L^{p}\left(E_{n}\right)$ norm as $t \rightarrow 0$, and

$$
\|F\|_{p}=\lim _{t \rightarrow 0}\|F(x ; t)\|_{p}=\|F(x ; 0)\|_{p}
$$

They also showed that if $p=(n-1) / n$, then the nontangential limit exists for almost every $x \in E_{n}$.

The Littlewood-Paley $g$-function for the half-space is defined by

$$
\begin{aligned}
& g(f)(x)=g(u)(x)=\left(\int_{0}^{\infty} t|\nabla u(x ; t)|^{2} d t\right)^{1 / 2}, \\
& g(F)(x)=\left(\frac{1}{n+1} \sum_{j=1}^{n+1} g^{2}\left(u_{j}\right)(x)\right)^{1 / 2} .
\end{aligned}
$$


For the Lusin $s$-function, the cone inside the unit sphere is replaced by an open cone $W_{\delta}(x), 0<\delta<\infty$, consisting of all points $(y ; t)$ such that $|x-y|<\delta t$.

The Lusin $s$-function for the half-space is then defined by

$$
\begin{aligned}
& s(f)(x)=s(u)(x)=\left(\iint_{W_{\delta}(x)} \frac{|\nabla u(y ; t)|^{2}}{t^{n-1}} d t d y\right)^{1 / 2}, \\
& s(F)(x)=\left(\frac{1}{n+1} \sum_{j=1}^{n+1} s^{2}\left(u_{j}\right)(x)\right)^{1 / 2} .
\end{aligned}
$$

It is known [12, pp. 447, 462] that, for any $u(x ; t)$ harmonic in $t>0, g(u)(x)$ $\leqq A_{\delta} s(u)(x)$, and so

$$
g(f)(x) \leqq A_{\delta} s(f)(x), \quad g(F)(x) \leqq A_{\delta} s(F)(x) .
$$

We now state the half-space analogues of Lemma 5 and of Theorems 1 and 2.

LeMMA 9. Suppose that $F \in H^{p}\left(E_{n} \times(0, \infty)\right), p>(n-1) / n$, and let

$$
N(F)(x)=N_{\delta}(F)(x)=\sup \left\{|F(y ; t)|:(y ; t) \in W_{\delta}(x)\right\} .
$$

Then

$$
\|N(F)\|_{p} \leqq A_{p, \delta}\|F\|_{p} .
$$

THEOREM 3. If $F \in H^{p}\left(E_{n} \times(0, \infty)\right)$, $(n-1) / n<p<\infty$, then $\|g(F)\|_{p} \leqq A_{p}\|F\|_{p}$ and $\|s(F)\|_{p} \leqq A_{p, \delta}\|F\|_{p}$.

THEOREM 4. If $f \in L^{p}\left(E_{n}\right), 1<p<\infty$, then $\|g(f)\|_{p} \leqq A_{p}\|f\|$ and $\|s(f)\| \leqq A_{p, \delta}\|f\|$.

Lemma 9 is an immediate consequence of two lemmas which appear in [13, Lemmas (3.8) and (3.14)]. As was mentioned in the introduction, Theorem 4 has been proved by Stein [12]. In $\S 7$, we shall show how Lemma 1 may be employed in order to obtain Theorem 3 for $(n-1) / n<p \leqq 2$. Since the extension to $p>2$ then proceeds in essentially the same way as that used in $\$ 5$ for the unit sphere, we shall not present it. The case $p>1$ of Theorem 3 may also be obtained by applying Theorem 4 to each component of $F$.

It should be noted that we may obtain a new proof of Theorem 4 for $1<p \leqq 2$ by proceeding along the same lines as in the proof of Theorem 3, replacing $F$ by $f$ and the various lemmas concerning systems of conjugate harmonic functions by the analogous lemmas for single harmonic functions.

7. Proof of Theorem 3; case when $(n-1) / n<p \leqq 2$. In view of (6.1), it suffices to prove that

$$
\|s(F)\|_{p} \leqq A_{p, \delta}\|F\|, \quad(n-1) / n<p \leqq 2 .
$$

Setting $F_{\varepsilon}(x ; t)=F(x ; t+\varepsilon), \varepsilon>0$, and proceeding as in the proof of Theorem 1 given in $\$ 4$ for $(n-2) /(n-1)<p \leqq 2$, we find that it is enough to prove (7.1) with 
$F(x ; t)$ replaced by $F_{\varepsilon}(x ; t)$. In the proof $F(x ; t)$ will denote $F_{\varepsilon}(x ; t)$.

For any two positive numbers $a$ and $b$, let $d=a+\delta b$ and

$$
h(x ; t)=\frac{b}{\pi} \cos ^{2} \frac{\pi r}{2 d} \sin \frac{\pi t}{b},
$$

where $r=|x|$. We note that

$$
\lim _{a \rightarrow \infty} h(x ; t)=\frac{b}{\pi} \sin \frac{\pi t}{b}, \quad \lim _{b \rightarrow \infty} \frac{b}{\pi} \sin \frac{\pi t}{b}=t,
$$

and $h(x ; t) \geqq 0$ whenever $0 \leqq t \leqq b$. Put

$$
\begin{aligned}
N(x) & =\sup _{(y: t) \in W_{\delta}(x)}|F(y ; t)|, \quad W(x)=W_{\delta}(x), \quad W(x ; b)=W(x) \cap\{(y ; t): t \leqq b\}, \\
c & =c(d)=\sup _{|x| \leqq \delta t-d}|F(x ; t)|^{2}, \quad w(x ; t)=w(F(x ; t), p, a, b)=\left(|F(x ; t)|^{2}+c\right)^{p / 2} .
\end{aligned}
$$

Then $0<c(d) \leqq N^{2}(x)$ and $w(x ; t) \leqq 2^{p / 2} N^{p}(x)$ whenever $|x| \leqq d$.

Letting

$$
S^{2}(x ; a, b)=\iint_{W(x ; b)} \frac{h(y ; t) \sum_{k=1}^{n+1}\left|\nabla u_{k}(y ; t)\right|^{2}}{(n+1) t^{n}} d t d y
$$

and applying Lemma 1 with $n$ replaced by $n+1$, it is easy to see that

$$
S^{2}(x ; a, b) \leqq A_{p} N^{2-p}(x) \iint_{W(x ; b)} \frac{h(y ; t) \Delta w(y ; t)}{t^{n}} d t d y
$$

whenever $|x| \leqq a$. Hence, using Hölder's inequality,

$$
\begin{aligned}
\int_{|x| \leqq a} S^{p}(x ; a, b) d x & \\
& \leqq A_{p}\|N\|_{p}^{p(2-p) / 2}\left(\int_{|x| \leqq a} \iint_{W(x ; b)} \frac{h(y ; t) \Delta w(y ; t)}{t^{n}} d t d y d x\right)^{p / 2} .
\end{aligned}
$$

Now let $\chi_{x}(y ; t)$ be the characteristic function of $W(x)$. Then

$$
\begin{aligned}
\int_{|x| \leqq a} \iint_{\mathbb{W}(x ; b)} \frac{h(y ; t) \Delta w(y ; t)}{t^{n}} d t d y d x \\
\quad=\int_{|x| \leqq a} \int_{|y| \leqq d} \int_{0}^{b} \frac{\chi x(y ; t) h(y ; t) \Delta w(y ; t)}{t^{n}} d t d y d x \\
\leqq A_{\delta} \int_{|y| \leqq d} \int_{0}^{b} h(y ; t) \Delta w(y ; t) d t d y
\end{aligned}
$$

since

$$
\int_{|x| \leqq a} \chi_{x}(y ; t) d x \leqq \int_{E_{n}} \chi_{x}(y ; t) d x=\int_{|x| \leqq \delta t} d x=A_{\delta} t^{n}
$$


Assuming temporarily that $n \geqq 2$ and employing cylindrical coordinates, we have

$$
\begin{aligned}
\int_{|x| \leqq d} \int_{0}^{b} h(x ; t) & \Delta w(x ; t) d t d x \\
= & \int_{|x| \leqq d} \int_{0}^{b} h(x ; t) \frac{\partial^{2} w}{\partial t^{2}} d t d x+\int_{\partial \Sigma} \int_{0}^{d} \int_{0}^{b} h(x ; t) \frac{\partial}{\partial r}\left(r^{n-1} \frac{\partial w}{\partial r}\right) d t d r d \theta \\
& +\sum_{j=1}^{n-1} \int_{Q} \int_{0}^{d} \int_{0}^{b} h(x ; t) \frac{\partial}{\partial t_{j}}\left(\frac{v}{v_{j}^{2}} \frac{\partial w}{\partial t_{j}}\right) d t d r d t_{1} \cdots d t_{n-1} \\
= & P_{t}+P_{r}+\sum_{j=1}^{n-1} P_{j},
\end{aligned}
$$

where $w=w(x ; t), r=|x|, x=r \theta$, and $Q, d \theta, v, v_{1}, \ldots, v_{n-1}$ are as defined in $\S 2$.

Consider $P_{t}$ first. Integration by parts with respect to $t$ yields

$$
\int_{0}^{b} \sin \frac{\pi t}{b} \frac{\partial^{2} w}{\partial t^{2}} d t=\left[-\frac{\pi}{b} w \cos \frac{\pi t}{b}\right]_{0}^{b}-\left(\frac{\pi}{b}\right)^{2} \int_{0}^{b} w \sin \frac{\pi t}{b} d t \leqq \frac{A_{p}}{b} N^{p}(x)
$$

whenever $|x| \leqq d$, and so

$$
P_{t} \leqq A_{p} \int_{|x| \leqq d} N^{p}(x) \cos ^{2} \frac{\pi r}{2 d} d x \leqq A_{p}\|N\|_{p}^{p}
$$

Considering $P_{r}$ next, we find that

$$
\int_{0}^{d} \cos ^{2} \frac{\pi r}{d} \frac{\partial}{\partial r}\left(r^{n-1} \frac{\partial w}{\partial r}\right) d r \leqq \frac{\pi^{2}}{2 d^{2}} \int_{0}^{d} r^{n-1} w d r
$$

and so $\left(d x=r^{n-1} d r d \theta\right)$

$$
P_{r} \leqq \frac{b \pi}{2 d^{2}} \int_{\partial \Sigma} \int_{0}^{d} \int_{0}^{b} r^{n-1} w \sin \frac{\pi t}{b} d t d r d \theta \leqq \frac{b^{2} A_{p}}{d^{2}}\|N\|_{p}^{p} \leqq A_{p, \delta}\|N\|_{p}^{p}
$$

Now consider $P_{j}$. An integration with respect to $t_{j}$ yields

$$
\int_{Q} \frac{\partial}{\partial t_{j}}\left(\frac{v}{v_{j}^{2}} \frac{\partial w}{\partial t_{j}}\right) d t_{1} \cdots d t_{n-1}=0, \quad j=1,2, \ldots, n-1,
$$

because

$$
\left[\sin ^{n-j-1} t_{j} \frac{\partial w}{\partial t_{j}}\right]_{t_{j}=0}^{t_{j}=\pi}=0, j<n-1
$$

and, due to periodicity,

$$
\left[\frac{\partial w}{\partial t_{n-1}}\right]_{t_{n-1}=0}^{t_{n-1}=2 \pi}=0 .
$$

Therefore, $P_{j} \equiv 0, j=1,2, \ldots, n-1$.

Combining these estimates, we find that

$$
\int_{|x| \leqq d} \int_{0}^{b} h(x ; t) \Delta w(x ; t) d t d x \leqq A_{p, \delta}\|N\|_{p .}^{p}
$$


If we now let $a$ and $b$ approach $\infty$ and apply Fatou's lemma, then it follows from (7.2), (7.3), (7.4), and Lemma 9 that

$$
\|s(F)\|_{p} \leqq A_{p, \delta}\|N\|_{p}^{(2-p) / 2}\|N\|_{p}^{p / 2} \leqq A_{p, \delta}\|F\|_{p}
$$

which is the desired result. For $n=1$, we use the cartesian form of $\Delta$ and proceed as above.

REMARKS. A simpler proof yields Theorems 2 and 4 for $1<p \leqq 2$. A decomposition of $f$ into its positive and negative parts reduces the problem to nonnegative functions. Then $u$, the Poisson integral of $f \geqq 0$, is strictly positive and so in place of Lemma 2 we may use the identity

$$
\Delta\left(u^{2}\right)=\frac{2}{p(p-1)} u^{2-p} \Delta\left(u^{p}\right)
$$

in which, since $u>0$, no singularities appear.

We note also that Theorems $1-4$ may be obtained for $p \geqq 4$ by using a standard conjugacy argument to pass from the case $1<p \leqq 2$ to $p \geqq 4$ (see Stein [12, p. 455] and Zygmund [17, Vol. II, p. 212]).

Added. E. M. Stein [Intégrales singulières et fonctions différentiables de plusiers variables (Notes), Faculté des Sciences d'Orsay, 1967] has independently utilized the identity (7.5) to obtain Theorem 4 and the analogous result for the function $g_{\sigma}^{*}(f)$ of Littlewood-Paley and Zygmund. Also, in Classes $H^{p}$ et multiplicateurs: Cas n-dimensionnel [C. R. Acad. Sci. Paris 264 (1967); Série A, 107-108, Proposition 2] he announced (without proof) the corresponding $H^{p}\left(E_{n} \times(0, \infty)\right), p>(n-1) / n$, result for the function $g_{\sigma}^{*}(F)$ (Stein denotes it by $S_{\lambda}^{*}(F)$ ). It is not difficult to see that Stein's Proposition 2 and the corresponding results for the class $H^{p}(\Sigma)$ and the $H^{p}$ class constructed by Muckenhoupt and Stein [9, p. 45] can be obtained by the method introduced in this paper.

\section{REFERENCES}

1. N. Aronszajn and K. T. Smith, Functional spaces and functional completion, Ann. Inst. Fourier (Grenoble) 6 (1955), 125-185.

2. A. P. Calderon, On the behavior of harmonic functions at the boundary, Trans. Amer. Math. Soc. 68 (1950), 47-54.

3. - Commutators of singular integral operators, Proc. Nat. Acad. Sci. U.S.A. 53 (1965), 1092-1099.

4. G. Gasper, Jr., On the Littlewood-Paley and Lusin functions in higher dimensions, Proc. Nat. Acad. Sci. U.S.A. 57 (1967), 25-28.

5. G. H. Hardy and J. E. Littlewood, A maximal theorem with function-theoretic applications, Acta Math. 54 (1930), 81-116.

6. J. E. Littlewood and R. E. A. C. Paley, Theorems on Fourier series and power series, Part I, J. London Math. Soc. 6 (1931), 230-233; Part II, Proc. London Math. Soc. 42 (1937), 52-89; Part III, ibid. 43 (1937), 105-126.

7. N. Lusin, Sur une propriété des fonctions à carré sommable, Bull. Calcutta Math. Soc. 20 (1930), 139-154.

8. J. Marcinkiewicz and A. Zygmund, $A$ theorem of Lusin, Duke Math. J. 4 (1938), 473-485. 
9. B. Muckenhoupt and E. M. Stein, Classical expansions and their relation to conjugate harmonic functions, Trans. Amer. Math. Soc. 118 (1965), 17-92.

10. I. Privaloff, Sur certaines classes de fonctions subharmoniques et leur représentation analytique, Dokl. Akad. Nauk. SSSR 2 (1938), 191-220.

11. K. T. Smith, A generalization of an inequality of Hardy and Littlewood, Canad. J. Math. 8 (1956), 157-170.

12. E. M. Stein, On the functions of Littlewood-Paley, Lusin, and Marcinkiewicz, Trans. Amer. Math. Soc. 88 (1958), 430-466.

13. E. M. Stein and G. Weiss, On the theory of harmonic functions of several variables, I. Acta Math. 103 (1960), 25-62.

14. C. de la Vallée Poussin, Propriétés des fonctions harmoniques dans un domaine ouvert limité par des surfaces à courbure bornée, Ann. Scuola Norm. Sup. Pisa 2 (1933), 167-197.

15. D. Waterman, On functions analytic in a half-plane, Trans. Amer. Math. Soc. 81 (1956), 167-194.

16. A. Zygmund, Proof of a theorem of Littlewood and Paley, Bull. Amer. Math. Soc. 51 (1945), 439-446.

17. —, Trigonometric series, Cambridge Univ. Press, New York, 1959.

WAyNe State University,

Detrott, Michigan

UNIVERSTTY OF WISCONSIN,

Madison, Wisconsin 